\title{
GRAPH THEORETIC BOUND ON NUMBER OF A.C.I.M. FOR RANDOM TRANSFORMATION
}

\author{
PAWEL GÓRA
}

(Communicated by Kenneth R. Meyer)

\begin{abstract}
Upper and lower bounds are given for the number of ergodic absolutely continuous invariant measures for a general random map. They are based on a communication graph describing the interaction of deterministic transformations used to define the random one. In particular, the case of piecewise expanding transformations is discussed.
\end{abstract}

\section{INTRODUCTION}

A random transformation $R$ induced by two deterministic transformations $\tau_{1}$ and $\tau_{2}$ is a Markov process that with given probabilities applies either $\tau_{1}$ or $\tau_{2}$ (see $\S 2$ for precise definitions). $R$ can be a mathematical model of a process whose behaviour depends on a randomly changing environment.

The general problem we partially consider in this paper is the examination of the properties of absolutely continuous invariant measures (a.c.i.m.) for $R$, given information about deterministic transformations used to define $R$.

Our results can be most successfully applied in the case of a random transformation induced by piecewise expanding transformation. The literature concerning those transformations is very vast, e.g., our references [1-8]. In particular, there are precise estimates of the number of absolutely continuous invariant measures for such transformations $[6,4,1]$. For random transformations there are only partial results available. In [8] the case when one of the deterministic transformations has a unique a.c.i.m. is studied, and in [1] the estimates are given in the case of a random transformation induced by piecewise linear Markov expanding transformations with a common defining partition.

In our paper we consider random maps in the general situation. Our main result, Theorem 1 , generalizes results of $[8,1]$. Theorem 3 is an application of Theorems 1 and 2 to the case of piecewise expanding transformations.

Received by the editors December 1, 1990 and, in revised form, March 4, 1991. 1991 Mathematics Subject Classification. Primary 28A65; Secondary 58F11. 
We present a number of examples. In particular, Example 4 shows that $R$ can have a.c.i.m. even if neither of $\tau_{1}, \tau_{2}$ has one. Example 5 presents an opposite phenomenon, both $\tau_{1}$ and $\tau_{2}$ have a.c.i.m., but $R$ does not have any.

\section{Definitions AND Notation}

Let $(X, m)$ be a measurable space with a probability measure $m$. Let $\tau$ : $X \rightarrow X$ be a measurable nonsingular transformation, $\tau_{*} m \ll m$. A measure $\mu$ is $\tau$-invariant if

$$
\mu(A)=\mu\left(\tau^{-1}(A)\right),
$$

for any measurable set $A$. We will restrict our attention to invariant measures that are absolutely continuous with respect to $m$, a.c.i.m.

Let $\mu_{1}, \ldots, \mu_{k}$ be ergodic a.c.i.m. for $\tau$. Let

$$
A_{i}=\operatorname{supp}\left(\mu_{i}\right), \quad i=1, \ldots, k,
$$

be disjoint measurable sets such that $\mu_{i}\left(A_{i}\right)=1$ and $\mu_{i}\left(A_{j}\right)=0$, for $i \neq j$. Let

$$
\mathscr{U}_{i}=\bigcup_{j=0}^{\infty} \tau^{-j}\left(A_{i}\right)
$$

for $i=1, \ldots, k . \mathscr{U}_{i}$ is called a basin of attraction of measure $\mu_{i}$.

Let $\tau_{1}, \tau_{2}$ be two transformations as above, with a.c.i.m. $\mu_{1}^{1}, \ldots, \mu_{k(1)}^{1}$ and $\mu_{1}^{2}, \ldots, \mu_{k(2)}^{2}$ respectively. Sets $A_{i}^{1}, \mathscr{U}_{i}^{1}, i=1, \ldots, k(1)$, and $A_{i}^{2}, \mathscr{U}_{i}^{2}, i=$ $1, \ldots, k(2)$, are defined as above.

We define the random transformation $R=R\left(\tau_{1}, \tau_{2}, \alpha, \beta\right)$ as a Markov process with transition function

$$
P(x, A)=\alpha \chi_{A}\left(\tau_{1}(x)\right)+\beta \chi_{A}\left(\tau_{2}(x)\right),
$$

for $\alpha, \beta>0, \alpha+\beta=1$. Intuitively it sends a point $x$ to $\tau_{1}(x)$ with probability $\alpha$ and to $\tau_{2}(x)$ with probability $\beta$.

We are interested in a number of ergodic absolutely continuous measures invariant for $R$. It is easy to see that $\mu$ is $R$-invariant iff

$$
\mu(A)=\alpha \mu\left(\tau_{1}^{-1}(A)\right)+\beta \mu\left(\tau_{2}^{-1}(A)\right),
$$

for any measurable set $A$.

The estimates we will prove depend on the following transition graph $G_{R}$ : its vertices are sets $\mathscr{U}_{i}{ }^{1}, i=1, \ldots, k(1)$, and $\mathscr{U}_{i}{ }^{2}, i=1, \ldots, k(2)$, and we draw an arrow from $\mathscr{U}_{i}^{s}$ to $\mathscr{U}_{j}^{t}$ iff

$$
m\left(\mathscr{U}_{j}^{t} \cap A_{i}^{s}\right)>0
$$

for $t \neq s, s, t \in\{1,2\}, 1 \leq i, j \leq \max (k(1), k(2))$. We also assume there is an arrow from any vertex to itself. 
Example 1. Let us consider the situation in Figure 1.

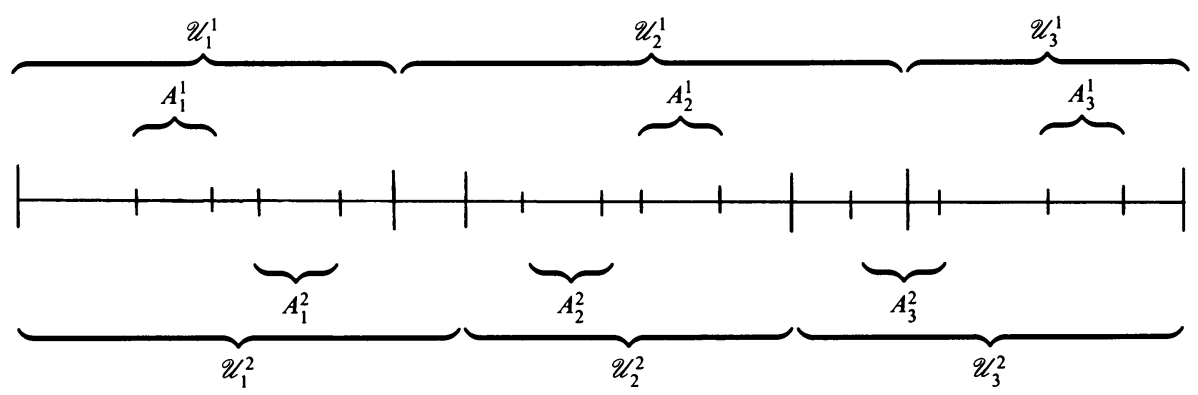

Figure 1

Graph $G_{R}$ looks as follows:

$$
\begin{aligned}
& \mathscr{U}_{1}^{1} \rightleftarrows \mathscr{U}_{1}^{2} \\
& \mathscr{U}_{2}^{1} \rightleftarrows \mathscr{U}_{2}^{2} \\
& \uparrow \\
& \mathscr{U}_{3}^{2} \rightleftarrows \mathscr{U}_{3}^{1}
\end{aligned}
$$

We define an ergodic component of $G_{R}$ as a family $\left\{\mathscr{U}_{q_{1}}^{s_{1}}, \ldots, \mathscr{U}_{q_{k}}^{s_{k}}\right\}$ such that for any sets $\mathscr{U}_{q}^{s}$ and $\mathscr{U}_{p}^{t}$ in this family, there exists a path of arrows in $G_{R}$ leading from $\mathscr{U}_{q}^{s}$ to $\mathscr{U}_{p}^{t}$ and there are no arrows going from sets of the family to the sets outside the family.

We define a basin of an ergodic component of $G_{R}$ as a family of all sets $\mathscr{U}_{q}^{s}$ such that there exists a path of arrows in $G_{R}$ leading from $\mathscr{U}_{q}^{s}$ to a set in this ergodic component.

We will denote the number of ergodic components of $G_{R}$ by $M\left(G_{R}\right)$. By $\mathscr{W}_{1}, \ldots, \mathscr{W}_{M\left(G_{R}\right)}$ we denote unions of sets in basins of ergodic components of $G_{R}$. If $\left\{\mathscr{U}_{q_{1}}^{s_{1}}, \ldots, \mathscr{U}_{q_{k}}^{s_{k}}\right\}$ is an ergodic component of $G_{R}$ such that $\mathscr{W}_{i}$ is the union of sets in its basin, we define

$$
\mathscr{V}_{i}=\bigcup_{i=1}^{k} A_{q_{i}}^{s_{i}},
$$

for $i=1, \ldots, M\left(G_{R}\right)$.

In Example $1, M\left(G_{R}\right)=2, \mathscr{W}_{1}=\mathscr{U}_{1}^{2}$, and $\mathscr{W}_{2}=\mathscr{U}_{2}^{1} \cup \mathscr{U}_{3}^{1}$.

We will also use another graph $g_{R}$. Its vertices are sets $\mathscr{W}_{1}, \ldots, \mathscr{W}_{M\left(G_{R}\right)}$. We connect sets $\mathscr{W}_{i}$ and $\mathscr{W}_{j}$ by a line iff

$$
m\left(\mathscr{W}_{i} \cap \mathscr{W}_{j}\right)>0 \text {. }
$$

In Example $1, g_{R}$ is

$$
\mathscr{W}_{1} \longrightarrow \mathscr{W}_{2} \text {. }
$$

We will denote the number of connected components of $g_{R}$ by $m\left(g_{R}\right)$.

Since most of our examples are piecewise expanding transformations of an interval, we review here some basic definitions and results regarding these maps. 
Transformation $\tau:[a, b] \rightarrow[a, b]$ is called piecewise monotonic if there exist $a=a_{0}<a_{1}<\cdots<a_{N}=b$ such that $\tau_{\mid\left(a_{i-1}, a_{i}\right)}$ is monotonic, $i=$ $1, \ldots, N$. If, moreover, $\inf \left(\left|\tau^{\prime}(x)\right|\right)>1$ on these intervals $, i=1, \ldots, N$, then $\tau$ is called a piecewise expanding map. It is known that a piecewise expanding map $\tau$, under some additional assumptions on continuity of $\tau_{\mid\left(a_{i-1}, a_{i}\right)}^{\prime}[2,3,5,7]$, has invariant measures absolutely continuous with respect to Lebesgue measure and that the union of basins of attraction of these measures is the whole interval $[a, b]$. The same facts are true for random maps induced by two piecewise expanding maps [8].

The main tool in exploring properties of piecewise monotonic maps is the Perron-Frobenius operator on $L^{1}([a, b], m)$,

$$
\left(P_{\tau} f\right)(x)=\sum_{i=1}^{N} \frac{f\left(\tau_{i}^{-1}(x)\right)}{\left|\tau^{\prime}\left(\tau_{i}^{-1}(x)\right)\right|},
$$

where $\tau_{i}^{-1}$ is the $i$ th branch of $\tau^{-1}$. A function $f$ satisfies $P_{\tau} f=f$ iff $f$ is a density of a.c.i.m. for $\tau$.

A piecewise monotonic transformation $\tau$ is called Markov if

$$
\tau\left(\left\{a_{0}, a_{1}, \ldots, a_{N}\right\}\right) \subset\left\{a_{0}, a_{1}, \ldots, a_{N}\right\} .
$$

If, moreover, it is linear on intervals $\left[a_{i-1}, a_{i}\right], i=1, \ldots, N$, we can consider $P_{\tau}$ restricted to the space of functions constant on these intervals. We will call this restriction a Perron-Frobenius matrix $\mathbf{P}_{\tau}$ of $\tau$. Any $\tau$-invariant density is constant on intervals $\left[a_{i-1}, a_{i}\right], i=1, \ldots, N$, and can be identified with a $\mathbf{P}_{\tau}$-invariant vector.

If $\tau_{1}$ and $\tau_{2}$ are two piecewise linear Markov monotonic transformations with the same defining partition and $R=R\left(\tau_{1}, \tau_{2}, \alpha, \beta\right)$, then

$$
\mathbf{P}_{R}=\alpha \mathbf{P}_{\tau_{1}}+\beta \mathbf{P}_{\tau_{2}} \text {. }
$$

\section{Main Results}

The main result of this note is Theorem 1, which gives an upper bound for the number of ergodic a.c.i.m. for a random map. It generalizes Theorem 3 of [1] .

Theorem 1. If $\bigcup_{i=1}^{M\left(G_{R}\right)} \mathscr{W}_{i}=X$ (m-a.e.), then the number of ergodic absolutely continuous $R$-invariant measures is at most $M\left(G_{R}\right)$, the number of ergodic components of $G_{R}$.

Let $\mu$ be an $R$-invariant absolutely continuous measure. Equality $(*)$ implies that if $\mu(B)>0$ then $\mu\left(\tau_{1}^{n}(B)\right)>0$ and $\mu\left(\tau_{2}^{n}(B)\right)>0$ for any measurable set $B$ and any positive integer $n$. We will use this repeatedly to prove the following facts.

Lemma 1. If $\mu\left(\mathscr{U}_{q}^{s}\right)>0$ for $s \in\{1,2\}$ and some $1 \leq q \leq k(s)$, then $A_{q}^{s} \subset \operatorname{supp}(\mu)$.

Proof. $\mu\left(\mathscr{U}_{q}^{s}\right)>0$ implies $\mu\left(A_{q}^{s}\right)>0$. Let $B \subset A_{q}^{s} \cap \operatorname{supp}(\mu), \mu(B)>$ $0, \mu_{q}^{s}(B)>0$. Since $\tau_{s \mid A_{q}^{s}}$ is $\mu_{q}^{s}$-ergodic, we have $A_{q}^{s}=\bigcup_{i=0}^{\infty} \tau_{s}^{i}(B)$ up to a set of $m$-measure zero. Thus $A_{q}^{s} \subset \operatorname{supp}(\mu)$. 
Lemma 2. If there is a path of arrows in $G_{R}$ leading from $\mathscr{U}_{q}^{s}$ to $\mathscr{U}_{w}^{z}, s, z \in$ $\{1,2\}, \quad 1 \leq q, w \leq \max (k(1), k(2))$, and $\mu\left(\mathscr{U}_{q}^{s}\right)>0$, then $A_{w}^{z} \subset \operatorname{supp}(\mu)$. Proof. We can assume there is a direct arrow from $\mathscr{U}_{q}^{s}$ to $\mathscr{U}_{w}^{z}$, as in the general case we would repeat our reasoning inductively. By Lemma 1 we have $A_{q}^{s} \subset \operatorname{supp}(\mu)$. By definition of $G_{R}$, we have $m\left(\mathscr{U}_{w}^{z} \cap A_{q}^{s}\right)>0$, so $\mu\left(\mathscr{U}_{w}^{z}\right)>0$. Again by Lemma 1 we get $A_{w}^{z} \subset \operatorname{supp}(\mu)$.

Proof of Theorem 1. Let $\mu_{1}$ and $\mu_{2}$ be two mutually singular a.c.i.m. for $R$ such that $\mu_{1}\left(\mathscr{W}_{i}\right)>0$ and $\mu_{2}\left(\mathscr{W}_{i}\right)>0$, for some $1 \leq i \leq M\left(G_{R}\right)$. Then we have $\mu_{1}\left(\mathscr{U}_{q}^{s}\right)>0$ and $\mu_{2}\left(\mathscr{U}_{p}^{t}\right)>0$, for some $\mathscr{U}_{q}^{s}, \mathscr{U}_{p}^{t} \subset \mathscr{W}_{i}$.

If $s=t$ and $q=p$, then Lemma 1 gives us

$$
A_{q}^{s} \subset \operatorname{supp}\left(\mu_{1}\right) \cap \operatorname{supp}\left(\mu_{2}\right),
$$

contradicting mutual singularity of $\mu_{1}$ and $\mu_{2}$.

If $\mathscr{U}_{q}^{s} \neq \mathscr{U}_{p}^{t}$, then since they both belong to the basin of the same ergodic component of $G_{R}$, there exists $\mathscr{U}_{w}^{z} \subset \mathscr{W}_{i}$ such that we can find paths of arrows in $G_{R}$ leading from $\mathscr{U}_{q}^{s}$ and $\mathscr{U}_{p}^{t}$ to $\mathscr{U}_{w}^{z}$. Lemma 2 gives us

$$
A_{w}^{z} \subset \operatorname{supp}\left(\mu_{1}\right) \cap \operatorname{supp}\left(\mu_{2}\right),
$$

again contradicting mutual singularity of $\mu_{1}$ and $\mu_{2}$.

This proves that the number of mutually singular a.c.i.m. for $R$ is not greater than $M\left(G_{R}\right)$, the number of ergodic components of $G_{R}$.

Corollary 1. For any $\mu$ a.c.i.m. for $R$, if $\mu\left(\mathscr{W}_{i}\right)>0$, then $\mathscr{V}_{i} \subset \operatorname{supp}(\mu)$.

Corollary 2. If $\tau_{1}$ and $\tau_{2}$ satisfy the assumptions of Theorem 1 , then the number of ergodic a.c.i.m. for $R$ is not greater than the minimum of the number of ergodic a.c.i.m. for $\tau_{1}$ and the number of ergodic a.c.i.m. for $\tau_{2}$.

In particular, if one of $\tau_{1}, \tau_{2}$ has the unique a.c.i.m. , then $R$ has the unique a.c.i.m.

Theorem 1 gives an upper bound for the number of $R$-ergodic a.c.i.m. Example 2 shows that the actual number of these measures can be smaller than $M\left(G_{R}\right)$.

Example 2. Let $\tau_{1}$ and $\tau_{2}$ be piecewise linear Markov expanding transformations as defined on Figures 2 and 3, respectively (see the next page).

We have $A_{1}^{1}=a \cup b, A_{2}^{1}=A_{2}^{2}=f \cup g, A_{1}^{2}=c \cup d, \mathscr{U}_{1}^{1}=a \cup b \cup c \cup d \cup e$, $\mathscr{U}_{2}^{1}=f \cup g, \mathscr{U}_{1}^{2}=a \cup b \cup c \cup d, \mathscr{U}_{2}^{2}=e \cup f \cup g$.

The graph $G_{R}$ is

$$
\begin{aligned}
& \mathscr{U}_{1}^{1} \rightleftarrows \mathscr{U}_{1}^{2} \\
& \mathscr{U}_{2}^{1} \rightleftarrows \mathscr{U}_{2}^{2} \\
& M\left(G_{R}\right)=2, \mathscr{W}_{1}=\mathscr{U}_{1}^{1}, \mathscr{W}_{2}=\mathscr{U}_{2}^{2} . \text { The graph } g_{R} \text { is } \\
& \mathscr{W}_{1} \rightleftarrows \mathscr{W}_{2},
\end{aligned}
$$

and $m\left(g_{R}\right)=1$. 


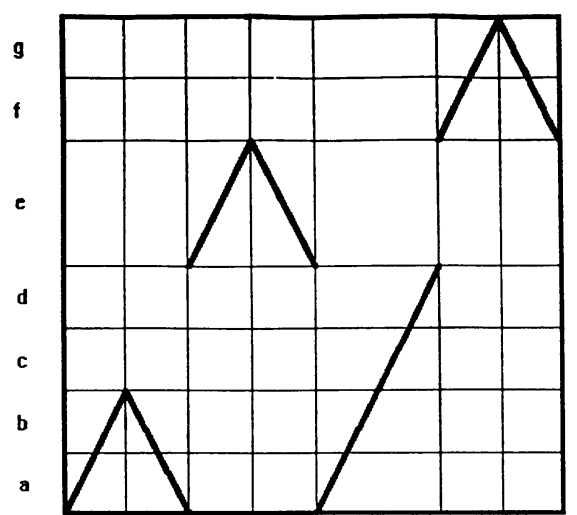

FIGURE 2

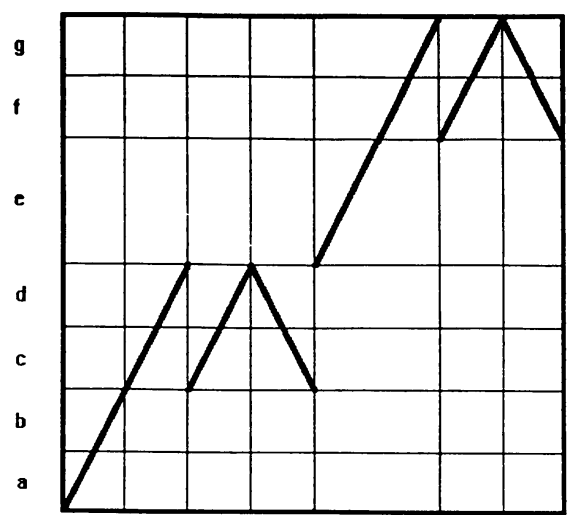

FIGURE 3

The Perron-Frobenius matrices of $\tau_{1}, \tau_{2}$, and $R=R\left(\tau_{1}, \tau_{2}, 1 / 2,1 / 2\right)$ are as follows:

$$
\begin{aligned}
& \mathbf{P}_{\tau_{1}}=\left(\begin{array}{ccccccc}
1 / 2 & 1 / 2 & 0 & 0 & 0 & 0 & 0 \\
1 / 2 & 1 / 2 & 0 & 0 & 0 & 0 & 0 \\
0 & 0 & 0 & 0 & 1 / 2 & 0 & 0 \\
0 & 0 & 0 & 0 & 1 / 2 & 0 & 0 \\
1 / 2 & 1 / 2 & 1 / 2 & 1 / 2 & 0 & 0 & 0 \\
0 & 0 & 0 & 0 & 0 & 1 / 2 & 1 / 2 \\
0 & 0 & 0 & 0 & 0 & 1 / 2 & 1 / 2
\end{array}\right), \\
& \mathbf{P}_{\tau_{2}}=\left(\begin{array}{ccccccc}
1 / 2 & 1 / 2 & 0 & 0 & 0 & 0 & 0 \\
0 & 0 & 1 / 2 & 1 / 2 & 0 & 0 & 0 \\
0 & 0 & 1 / 2 & 1 / 2 & 0 & 0 & 0 \\
0 & 0 & 1 / 2 & 1 / 2 & 0 & 0 & 0 \\
0 & 0 & 0 & 0 & 1 / 2 & 1 / 2 & 1 / 2 \\
0 & 0 & 0 & 0 & 0 & 1 / 2 & 1 / 2 \\
0 & 0 & 0 & 0 & 0 & 1 / 2 & 1 / 2
\end{array}\right), \\
& \mathbf{P}_{R}=\left(\begin{array}{ccccccc}
1 / 2 & 1 / 2 & 0 & 0 & 0 & 0 & 0 \\
1 / 4 & 1 / 4 & 1 / 4 & 1 / 4 & 0 & 0 & 0 \\
0 & 0 & 1 / 4 & 1 / 4 & 1 / 4 & 0 & 0 \\
0 & 0 & 1 / 4 & 1 / 4 & 1 / 4 & 0 & 0 \\
1 / 4 & 1 / 4 & 1 / 4 & 1 / 4 & 1 / 4 & 1 / 4 & 1 / 4 \\
0 & 0 & 0 & 0 & 0 & 1 / 2 & 1 / 2 \\
0 & 0 & 0 & 0 & 0 & 1 / 2 & 1 / 2
\end{array}\right) .
\end{aligned}
$$

The unique normalized invariant vector of $\mathbf{P}_{R}$ is $(0,0,0,0,0,4,4)$, so the number of a.c.i.m. for $R$ is $1<M\left(G_{R}\right)$.

Remark. It is not necessarily true that for $R$-ergodic a.c.i.m. $\mu, \mu\left(\mathscr{W}_{i}\right)>0$ implies $\mathscr{V}_{i}=\operatorname{supp}(\mu)$. A simple example can be produced by a small change in Example 2. If we redefine $\tau_{1}$ on interval $f$ in such a way that $\tau_{1}(f)=e$, then $R$ has the unique a.c.i.m. $\mu$ and $\operatorname{supp}(\mu)=X$.

Example 3 shows that sometimes $M\left(G_{R}\right)$ is the number of $R$-ergodic a.c.i.m.

Example 3. Let $\tau_{1}$ and $\tau_{2}$ be piecewise linear Markov expanding transformations as defined on Figures 4 and 5, respectively. 


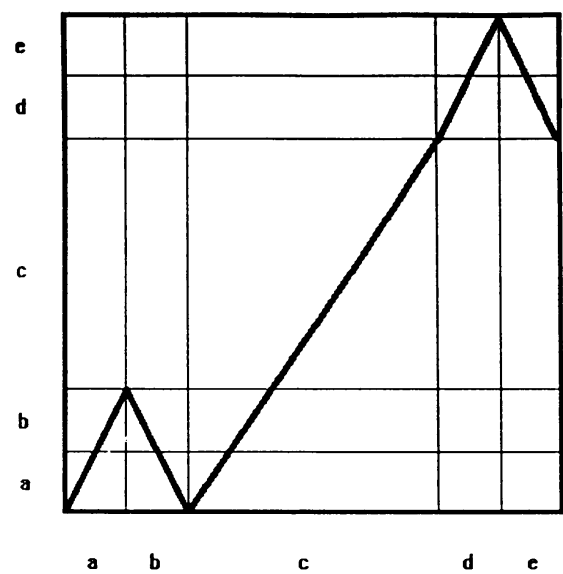

Figure 4

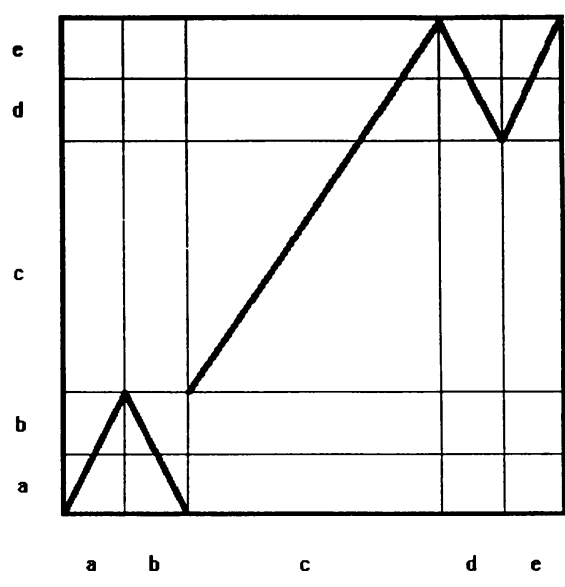

Figure 5

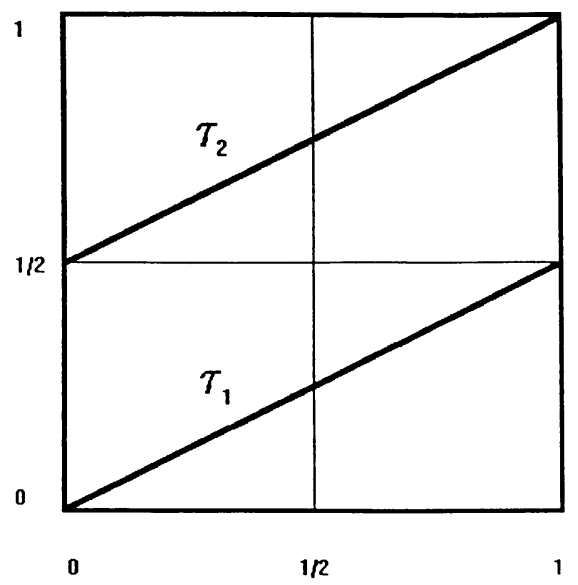

FIGURE 6

We have $A_{1}^{1}=A_{1}^{2}=a \cup b, A_{2}^{1}=A_{2}^{2}=d \cup e, \mathscr{U}_{1}^{1}=a \cup b \cup c, \mathscr{U}_{2}^{1}=d \cup e$, $\mathscr{U}_{1}^{2}=a \cup b, \mathscr{U}_{2}^{2}=c \cup d \cup e$.

The graph $G_{R}$ is

$$
\begin{aligned}
& \mathscr{U}_{1}^{1} \rightleftarrows \mathscr{U}_{1}^{2} \\
& \mathscr{U}_{2}^{1} \rightleftarrows \mathscr{U}_{2}^{2}
\end{aligned}
$$

$M\left(G_{R}\right)=2, \mathscr{W}_{1}=\mathscr{U}_{1}^{1}, \mathscr{W}_{2}=\mathscr{U}_{2}^{2}$. The graph $g_{R}$ is

$$
\mathscr{W}_{1} \longrightarrow \mathscr{W}_{2} \text {, }
$$

and $m\left(g_{R}\right)=1$. It is easy to prove that $R$ has two ergodic a.c.i.m. with supports $A_{1}^{1}$ and $A_{2}^{1}$.

In Example 4 we produce an a.c.i.m. "out of nothing," showing the importance of assumption $\bigcup_{i=0}^{M\left(G_{R}\right)} \mathscr{W}_{i}=X,(m$-a.e. $)$ in Theorem 1 .

Example 4. Let $\tau_{1}(x)=x / 2, \tau_{2}(x)=1 / 2+x / 2,0 \leq x \leq 1$ (see Figure 6). 
Neither transformation $\tau_{1}, \tau_{2}$ has an a.c.i.m. with respect to Lebesgue measure. On the other hand, it is easy to check that the random transformation $R=R\left(\tau_{1}, \tau_{2}, 1 / 2,1 / 2\right)$ leaves Lebesgue measure invariant.

Theorem 2 gives a lower bound on the number of a.c.i.m. for $R$.

Theorem 2. We assume that

$$
\begin{gathered}
X=\bigcup_{i=1}^{k(1)} \mathscr{U}_{i}^{1}=\bigcup_{i=1}^{k(2)} \mathscr{U}_{i}^{2}, \\
X=\bigcup_{i \geq 1} \mathscr{U}_{i}^{R},
\end{gathered}
$$

where $\mathscr{U}_{i}^{R}$ are basins of attraction of $R$-ergodic a.c.i.m.. Then the number of $R$-ergodic a.c.i.m. is at least $m\left(g_{R}\right)$, the number of connected subgraphs in $g_{R}$. Proof. Let $\mathscr{Y}_{i}, i=1, \ldots, m\left(g_{R}\right)$, be unions of sets $\mathscr{W}_{j}$ in connected subgraphs of $g_{R}$. By assumption $(* *)$ and the definition of $g_{R}$, we have

$$
\tau_{1}\left(\mathscr{Y}_{i}\right) \subset \mathscr{Y}_{i}, \quad \tau_{2}\left(\mathscr{Y}_{i}\right) \subset \mathscr{Y}_{i},
$$

$m$-a.e. for $1 \leq i \leq m\left(g_{R}\right)$. This means that any set $\mathscr{Y}_{i}$ is $R$-invariant. Assumption $(* * *)$ implies that, for any $1 \leq i \leq m\left(g_{R}\right)$, there is an a.c.i.m. $\mu_{i}$ for $R$ with $\mu_{i}\left(\mathscr{Y}_{i}\right)>0$. So we have at least $m\left(g_{R}\right)$ mutually singular a.c.i.m. for $R$.

Example 3 shows that the number of $R$-ergodic a.c.i.m. can be bigger than $m\left(g_{R}\right)$.

It may seem that the assumptions of Theorem 2 are unnecessarily strong. Example 5 shows that sometimes $(* *)$ does not imply $(* * *)$.

Example 5. Let $X=[0,1]$ and $m$ be Lebesgue measure. Let us define

and

$$
\tau_{1}(x)= \begin{cases}2 x, & \text { for } 0 \leq x \leq \frac{1}{4} \\ \frac{1}{2}-2\left(x-\frac{1}{4}\right), & \text { for } \frac{1}{4}<x \leq \frac{1}{2} \\ \frac{1}{4}-\frac{1}{2}\left(x-\frac{1}{2}\right), & \text { for } \frac{1}{2}<x \leq 1\end{cases}
$$

$$
\tau_{2}(x)=1-\left(\tau_{1}(1-x)\right)
$$

(see Figure 7). We have $A_{1}^{1}=[0,1 / 2], A_{1}^{2}=[1 / 2,1]$, and $\mathscr{U}_{1}^{1}=\mathscr{U}_{1}^{2}=[0,1]$. We will show that $R=R\left(\tau_{1}, \tau_{2}, 1 / 2,1 / 2\right)$ does not have any a.c.i.m.

The common Markov partition for $\tau_{1}$ and $\tau_{2}$ is infinite and consists of intervals $I_{-n}=\left[1 / 2^{n+1}, 1 / 2^{n}\right]$ and $I_{n}=\left[1-1 / 2^{n}, 1-1 / 2^{n+1}\right], n=1,2, \ldots$.

Let us assume that $R$ has an a.c.i.m. $\mu$. By Theorem 1 it is unique. Its density is constant on intervals $I_{-n}, I_{n}, n=1,2, \ldots$, and can be identified with the $\mathbf{P}_{R}$-invariant vector $\mathbf{v}=\left(\ldots, v_{-2}, v_{-1}, v_{1}, v_{2}, \ldots\right)$. By Corollary 1 , we have $[0,1] \subset \operatorname{supp}(\mu)$, so $v_{-n}, v_{n}>0, n=1,2, \ldots$.

$\mathbf{P}_{R}$ has the following positive elements:

$$
a_{-n-1,-n}=a_{-1,-n}=a_{1, n}=a_{n+1, n}=\frac{1}{4}
$$

and

$$
a_{n,-n-1}=a_{-n, n+1}=1
$$




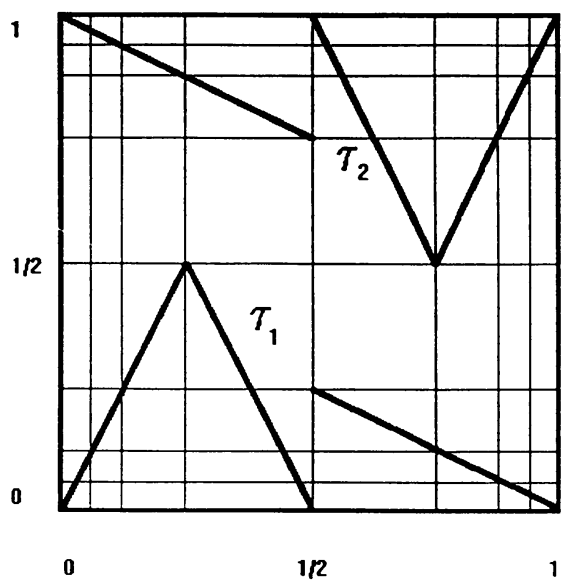

FIGURE 7

for $n=1,2, \ldots$. All other elements of $\mathbf{P}_{R}$ are equal to 0 .

$$
\begin{array}{ccccccccccc} 
& \ldots & I_{-4} & I_{-3} & I_{-2} & I_{-1} & I_{1} & I_{2} & I_{3} & I_{4} & \ldots \\
\vdots & \ddots & \vdots & \vdots & \vdots & \vdots & \vdots & \vdots & \vdots & \vdots & \\
I_{-5} & \ldots & \frac{1}{4} & 0 & 0 & 0 & 0 & 0 & 0 & 0 & \ldots \\
I_{-4} & \ldots & 0 & \frac{1}{4} & 0 & 0 & 0 & 0 & 0 & 0 & \ldots \\
I_{-3} & \ldots & 0 & 0 & \frac{1}{4} & 0 & 0 & 0 & 0 & 1 & \ldots \\
I_{-2} & \ldots & 0 & 0 & 0 & \frac{1}{4} & 0 & 0 & 1 & 0 & \ldots \\
\mathbf{P}_{-1} & \ldots & \frac{1}{4} & \frac{1}{4} & \frac{1}{4} & \frac{1}{4} & 0 & 1 & 0 & 0 & \ldots \\
I_{1} & \ldots & 0 & 0 & 1 & 0 & \frac{1}{4} & \frac{1}{4} & \frac{1}{4} & \frac{1}{4} & \ldots \\
I_{2} & \ldots & 0 & 1 & 0 & 0 & \frac{1}{4} & 0 & 0 & 0 & \ldots \\
I_{3} & \ldots & 1 & 0 & 0 & 0 & 0 & \frac{1}{4} & 0 & 0 & \ldots \\
I_{4} & \ldots & 0 & 0 & 0 & 0 & 0 & 0 & \frac{1}{4} & 0 & \ldots \\
I_{5} & \ldots & 0 & 0 & 0 & 0 & 0 & 0 & 0 & \frac{1}{4} & \ldots \\
& & & & & & & & &
\end{array}
$$

Since matrix $\mathbf{P}_{R}$ is centrally symmetric, vector $\mathbf{v}$ is also symmetric, i.e., $v_{-n}=v_{n}$, for $n=1,2, \ldots$. We will find $\mathrm{v}$ as a solution of the equation $\mathbf{v} \mathbf{P}_{R}=\mathbf{v}$.

The $I_{1}$ column gives the equation

$$
\frac{1}{4} v_{1}+\frac{1}{4} v_{2}=v_{1} \Rightarrow v_{2}=3 v_{1} \text {. }
$$

The $I_{2}$ column gives the equation (we use $v_{-1}=v_{1}$ )

$$
v_{1}+\frac{1}{4} v_{1}+\frac{1}{4} v_{3}=v_{2} \Rightarrow v_{3}=7 v_{1} \text {. }
$$

In general, the $I_{n}$ column, $n \geq 2$, gives (using $v_{-n}=v_{n}$ )

$$
v_{n-1}+\frac{1}{4} v_{1}+\frac{1}{4} v_{n+1}=v_{n} \text {. }
$$

Using standard methods of recurrent equations theory we get

$$
v_{n+1}-5 v_{n}+8 v_{n-1}-4 v_{n-2}=0, \quad n \geq 3,
$$


and the unique solution

$$
v_{n}=\left(2^{n}-1\right) v_{1}, \quad n=1,2, \ldots
$$

Thus

$$
\mu([0,1])=2 \sum_{n=1}^{\infty} v_{n} m\left(I_{n}\right)=2 \sum_{n=1}^{\infty}\left(2^{n}-1\right) v_{1} \frac{1}{2^{n+1}}=+\infty
$$

$\mu$ is an infinite ( $\sigma$-finite) measure with singularities of density at 0 and 1 .

This proves that $R$ does not have any finite a.c.i.m.

In the case of one- or higher-dimensional piecewise expanding transformations, Theorems 1 and 2 imply the following

Theorem 3. If $\tau_{1}$ and $\tau_{2}$ are piecewise expanding transformations satisfying some assumptions implying the existence of a.c.i.m. with respect to Lebesgue measure (e.g., piecewise $C^{1+\varepsilon}$ ), then the number of ergodic a.c.i.m. for $R=$ $R\left(\tau_{1}, \tau_{2}, \alpha, \beta\right)$ is between $m\left(g_{R}\right)$ and $M\left(G_{R}\right)$.

In particular, it is not greater than the number of ergodic a.c.im. for any of $\tau_{1}$ and $\tau_{2}$.

The results of this note can be easily generalized to the case of random transformation induced by more than two transformations.

\section{REFERENCES}

1. A. Boyarsky and W. Byers, A graph-theoretic bound on the number of independent absolutely continuous invariant measures, J. Math. Anal. Appl. 139 (1989), 139-151.

2. P. Collet and J.-P. Eckmann, Measures invariant under mappings of the unit interval, Regular and Chaotic Motions in Dynamic Systems, NATO ASI Series (G. Velo and A.S. Wightman eds.), Plenum Press, New York and London, 1983, pp. 233-265.

3. P. Góra and A. Boyarsky, Absolutely continuous invariant measures for piecewise expanding $C^{2}$ transformations in $R^{N}$, Israel J. Math. 67 (1989), 272-286.

4. P. Góra, A. Boyarsky, and H. Proppe, On the number of invariant measures for higher dimensional chaotic transformations, J. Statist. Phys. 62 (1991), 1000-1017.

5. A. Lasota and J. Yorke, On the existence of invariant measures for piecewise monotonic transformations, Trans. Amer. Math. Soc. 186 (1973), 481-486.

6. T.-Y. Li and J. A. Yorke, Ergodic transformations from an interval into itself, Trans. Amer. Math. Soc. 235 (1978), 183-192.

7. You-Shi Lou, The existence of absolutely continuous invariant measures for $C^{1+\varepsilon}$ Jabłonski transformations in $R^{N}$, Proc. Amer. Math. Soc. 109 (1990), 105-112.

8. S. Pelikan, Invariant densities for random maps of interval, Trans. Amer. Math. Soc. 281 (1984), 813-825.

Department of Mathematics and Statistics, Concordia University, loyola Campus, 7141 Sherbrooke Street West, Montreal, Quebec, H4B 1 R6 Canada 